\title{
Photonic crystal fiber technology for monolithic single-mode large mode area all-solid amplifier
}

\author{
Christensen, Simon Lønborg; Papior, Sidsel R.; Johansen, Mette M. ; Hauge, Jakob Milo; Weirich, \\ Johannes ; Jakobsen, Christian ; Michieletto, Mattia ; Bondu, Magalie; Triches, Marco ; Alkeskjold, \\ Thomas T.
}

Total number of authors:

11

Published in:

Proceedings of SPIE

Link to article, DOI:

10.1117/12.2506995

Publication date:

2019

Document Version

Publisher's PDF, also known as Version of record

Link back to DTU Orbit

Citation $(A P A)$ :

Christensen, S. L., Papior, S. R., Johansen, M. M., Hauge, J. M., Weirich, J., Jakobsen, C., Michieletto, M., Bondu, M., Triches, M., Alkeskjold, T. T., \& Lægsgaard, J. (2019). Photonic crystal fiber technology for monolithic single-mode large mode area all-solid amplifier. In Proceedings of SPIE (Vol. 10897). [108971L ] SPIE - International Society for Optical Engineering. Proceedings of SPIE - The International Society for Optical Engineering https://doi.org/10.1117/12.2506995

\section{General rights}

Copyright and moral rights for the publications made accessible in the public portal are retained by the authors and/or other copyright owners and it is a condition of accessing publications that users recognise and abide by the legal requirements associated with these rights.

- Users may download and print one copy of any publication from the public portal for the purpose of private study or research.

- You may not further distribute the material or use it for any profit-making activity or commercial gain

- You may freely distribute the URL identifying the publication in the public portal 


\section{Photonic crystal fiber technology for monolithic single-mode large mode area all-solid amplifier}

Simon L. Christensen, Sidsel R. Papior, Mette M. Johansen, Jakob M. Hauge, Johannes Weirich, et al.

Simon L. Christensen, Sidsel R. Papior, Mette M. Johansen, Jakob M. Hauge, Johannes Weirich, Christian Jakobsen, Mattia Michieletto, Magalie Bondu, Marco Triches, Thomas T. Alkeskjold, Jesper Lægsgaard, "Photonic crystal fiber technology for monolithic single-mode large mode area all-solid amplifier," Proc. SPIE 10897, Fiber Lasers XVI: Technology and Systems, 108971L (7 March 2019); doi: 10.1117/12.2506995

SPIE. Event: SPIE LASE, 2019, San Francisco, California, United States 


\title{
Photonic crystal fiber technology for monolithic single-mode large mode area all-solid amplifier
}

\author{
Simon L. Christensen ${ }^{\mathrm{a}, *}$, Sidsel R. Papior ${ }^{\mathrm{b}}$, Mette M. Johansen ${ }^{\mathrm{b}}$, Jakob M. Hauge ${ }^{\mathrm{a}}$, Johannes \\ Weirich $^{\mathrm{b}}$, Christian Jakobsen ${ }^{\mathrm{b}}$, Mattia Michieletto ${ }^{\mathrm{b}}$, Magalie Bondu ${ }^{\mathrm{b}}$, Marco Triches ${ }^{\mathrm{b}}$, Thomas T. \\ Alkeskjold $^{\mathrm{b}}$, and Jesper Lægsgaard ${ }^{\mathrm{a}}$ \\ aDTU Fotonik, Technical University of Denmark, Ørsteds Plads, DK-2800 Kgs. Lyngby, Denmark \\ ${ }^{b}$ NKT Photonics A/S, Blokken 84, DK-3460 Birkerød, Denmark \\ *Corresponding author: slon@fotonik.dtu.dk
}

\begin{abstract}
In this work, an all-solid Ytterbium doped double-clad single-mode (SM) large-mode-area (LMA) photonic crystal fiber (PCF) is characterized in a monolithic amplifier setup. The characterized all-solid PCF combines the SM operation of LMA PCFs while maintaining simple splicing of all-solid fibers. The fiber has been tested in a pulsed monolithic forward pumped amplifier system achieving excellent modal properties. The fiber is polarization maintaining and has a mode field diameter of approximately $24 \mu \mathrm{m}$. At optimal fiber length the output signal power of the amplifier was measured to $43 \mathrm{~W}$ limited by pump power with an optical to optical efficiency of $61 \%$. Polarization extinction ratios (PER) above $18 \mathrm{~dB}$ was observed for all fiber lengths. Simple splicing with splice losses $<0.2 \mathrm{~dB}$ and maintained polarization are reported using a standard Fujikura fusion splicer.
\end{abstract}

Keywords: Photonic crystal fiber, fiber amplifier, ultrafast, monolithic, single-mode, all-solid fiber, high power, largemode-area

\section{INTRODUCTION}

In the last two decades, fiber lasers and amplifiers have revolutionized the field of high power laser sources by achieving near diffraction limited modal properties for high power operation [1]. The long interaction length between the seed and active material allows for large gain. Another benefit of the geometry of a fiber is the large ratio between surface area and volume, which ensures excellent heat removal. Intensity-dependent non-linear effects such as Raman scattering and self-phase-modulation (SPM) have driven the development of larger core fibers to mitigate these effects. Single-mode (SM) operation of larger cores demands better precision on the index difference between the core and cladding, which is limited by the control of doping concentration for step-index fibers (SIF). Conventional photonic crystal fibers (PCF) use a periodic structure of air holes to define the guiding of light in the core [2]. The size of the air holes can be changed during drawing achieving excellent control of the effective index contrast. The control of the size of air holes can be used to fabricate SM large-mode-area (LMA) fiber amplifiers [3].

Monolithic amplifier systems have very attractive properties with regard to stability, simplicity and practical handling due to the elimination of free space optics. The simplest method of splicing fibers is by fusing the two fiber ends together using heat. For conventional PCF, fusion splicing can be achieved but will be more complex compared to solid fiber splicing due to the collapse of air holes [4]. The added complexity of the splice can be accepted for high-end monolithic amplifiers but will be unwanted in mid-range amplifiers where interfacing complexity and costs are crucial. An all-solid PCF has been developed and fabricated by NKT Photonics A/S to reduce splicing complexity while maintaining SM operation for LMA fibers. Even though all-solid PCF have been reported previously [5, 6], this fiber differentiates itself with simple interfacing, SM LMA operation, and low cost.

Fiber Lasers XVI: Technology and Systems, edited by Adrian L. Carter, Proc. of SPIE Vol. 10897, 108971L · (c) 2019 SPIE · CCC code: 0277-786X/19/\$18 · doi: 10.1117/12.2506995 
This paper is organized as follows: In section 2, the passive characterization of the fiber will be presented. This includes the geometry, splicing abilities, and modal properties of the fiber. The active characterization of the fiber in a pulsed monolithic setup is presented in section 3. A conclusion is given in section 4 .

\section{PASSIVE CHARACTERIZATION}

\subsection{Geometry of the fiber}

The fiber, named DC-250/30-PM-Yb-FUD, is fabricated at NKT Photonics A/S and has a core diameter of approximately $30 \mu \mathrm{m}$. The fiber has two claddings where the outer cladding consists of a low index polymer coating and the inner cladding consists of silica and doped silica. A high index polymer coats the fiber for protection against mechanical damage and results in an effective outer diameter of approximately $380 \mu \mathrm{m}$. The pump is guided in the inner cladding, which has a diameter of $250 \mu \mathrm{m}$. The core light is confined by low index inclusions in a hexagonal lattice and stress applying parts (SAP). The SAPs also cause polarization maintaining abilities by inducing a stress related birefringence in the core. The center of the inner cladding can be seen in figure 1 . The two black regions, seen on both sides of the core, are the SAPs, which induce birefringence.

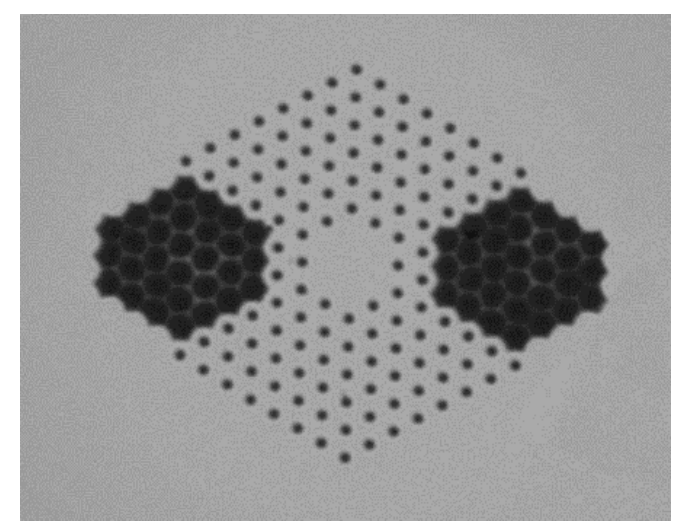

Figure 1: Cross sectional view of the all-solid photonic crystal fiber.

\subsection{Splicing abilities}

The loss of power and polarization extinction ratio (PER) through a splice are important for monolithic amplifiers. Low splice losses ensure efficient use of seed power and low heat generation in the splice. All splices presented have been performed with a Fujikura FSM 100p+ splicer using a standard program without any optimization.

In this work, the splice loss has been defined as the fundamental mode (FM) to FM loss through the splice. A sketch of the setup used to measure the splice loss can be seen in figure 2. The setup consists of an Ytterbium amplified spontaneous emission (ASE) source, lenses, a linear polarizer (LP), a half-wave-plate (HWP), and an optical spectrum analyzer (OSA). The polarized source is free-space coupled into the fiber under test (FUT). The coils of the fiber ensure only FM core light after the coil. After the second coil, the cladding light from the fiber is stripped and the core light is collected by a high numerical aperture (HNA) fiber. The power of collected light is measured by an OSA. 


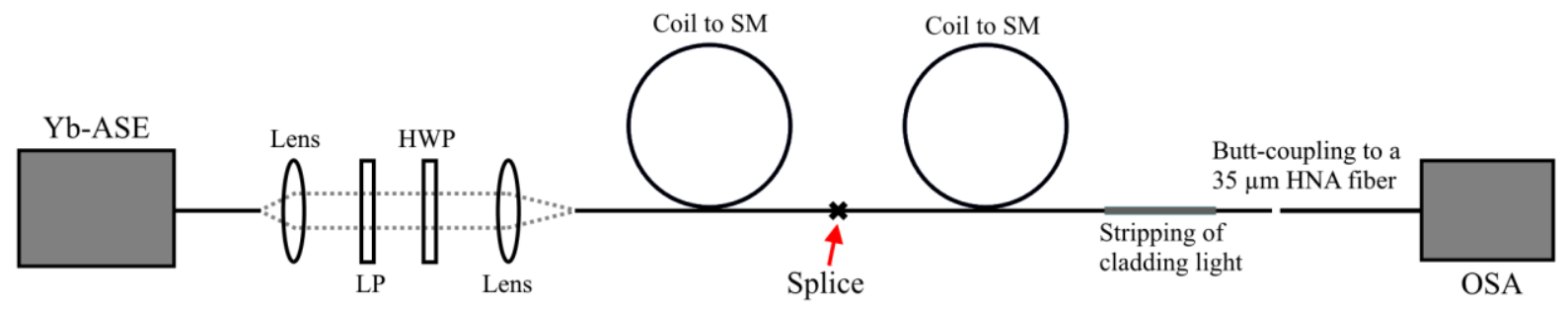

Figure 2: Sketch of setup for the splice loss measurement.

The measurement consisted of a reference spectrum where the fiber is in one piece, and a spectrum after the fiber was cleaved and spliced between the two coils. The splice loss was found by the difference of the two power spectra. The measured splice loss and error estimation can be seen in figure $3 \mathrm{a}$. The figure shows a splice loss below $0.2 \mathrm{~dB}$ including error estimation for all wavelengths between $1005 \mathrm{~nm}$ and $1200 \mathrm{~nm}$. The uncertainty on the splice loss measurement was estimated to $0.05 \mathrm{~dB}$ using a stability test and is represented in the figure by the shaded area. The error of $0.05 \mathrm{~dB}$ of the measurement system was most likely caused by temperature fluctuations, which would affect both the couplings and source. The splice loss measurement showed excellent splicing abilities of the all-solid fiber to itself with standard equipment and splice programs. The low splice loss showed that the all-solid fiber maintains the core guiding of the photonic crystal structure unlike conventional PCF where air holes collapse. The splice loss to other all-solid fibers is expected to mainly depend on the inherent mode mismatch of the two fibers since no structural changes to the fiber occurs.

Another important parameter of a splice is the ability to maintain the polarization of the core light. This is investigated using a slightly different setup using a LP and lenses on the output side and a shorter fiber. The PER was measured 10 $\mathrm{cm}$ after a splice and the splice was cut off and the PER was measured again. The measurement of PER can be seen in figure $3 b$, which shows high and stable PER for a wide span of wavelengths both before and after the splice. The difference in the measured PER is small and depends on wavelength. The two different output couplings most probably caused the difference in measured PER.

a)

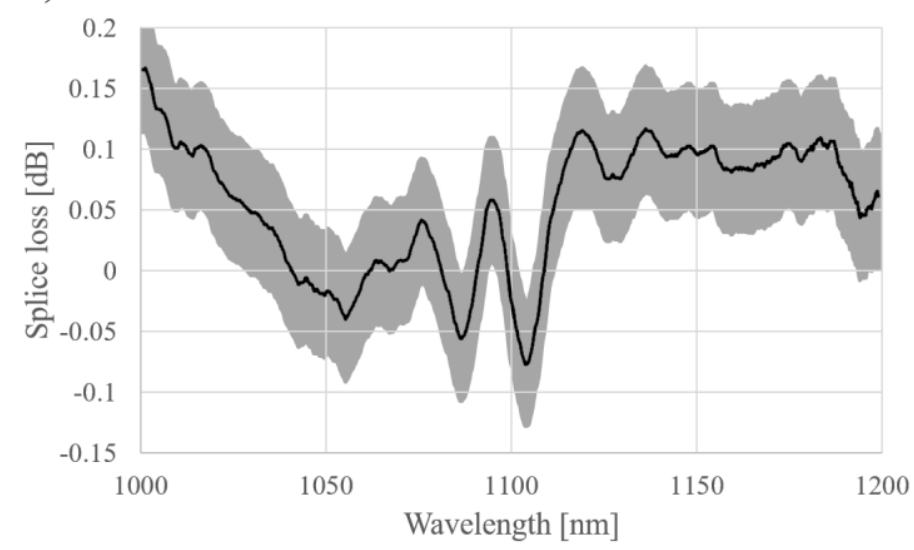

b)

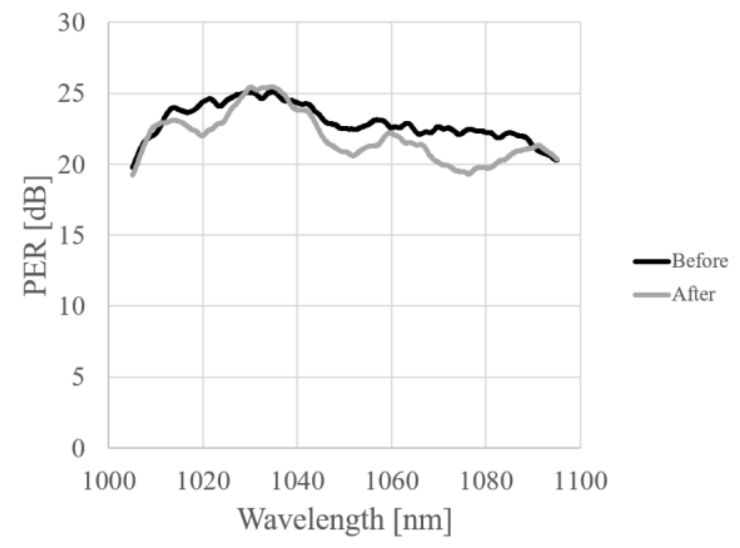

Figure 3: a) Measurement of the splice loss as a function of wavelength. The shaded area represent one standard deviation of the temporal changes of the system. b) Measurement of the PER before and after a splice. The loss and PER are not measured for the same splice. 


\subsection{Modal properties}

The modal properties determine the optimal configuration of the fiber in an active setup by ensuring small loss of the FM and high loss of higher order modes (HOM). The characterization of modal properties will consist of a bending loss measurement and a misalignment test investigating the modal properties of the FM and HOM, respectively.

The minimum coil diameter of the fiber is found measuring bend loss. The core power is measured for the slow and fast axis while reducing the coil diameter, as it can be seen in figure $4 \mathrm{a}$. The slow axis has a higher refractive index than the fast axis due to the birefringence of the fiber and the FM in the slow polarization is therefore better confined. For the FM polarized in the slow axis, the minimum coil diameter is approximately $19 \mathrm{~cm}$ and consequently, configurations with coil diameters below $19 \mathrm{~cm}$ are not efficient. The minimum coil of the FM polarized in fast axis does not have a clear cut-off coil diameter. The loss and regaining of core power is most likely caused by cladding-modes resonances, since the same features was observed in different measurements of the same fiber. The cladding mode resonances can cause coupling between core and cladding modes for specific coil diameters and wavelengths. At $25 \mathrm{~cm}$, a large drop in core power of the FM polarized in the fast axis is observed and the fiber will consequently be slightly polarizing for coil diameters from 19 to $25 \mathrm{~cm}$.

To ensure a stable output and diffraction limited beam quality, single-mode operation of the fiber is required. To investigate the modal properties of HOMs, a misalignment test has been performed. In a misalignment test, the input coupling beam is translated in the transverse plane to excite HOMs while the transmitted output field profile is observed. Figure $4 \mathrm{~b}$ shows the output field measured by an infrared nearfield camera when the input coupling is translated for a coil diameter of $22 \mathrm{~cm}$. The misalignment test shows no distortion of the output which means that all HOM have high losses and the fiber is effectively single-mode. The FM polarized in the slow axis has a mode field diameter (MFD) of approximately $24 \mu \mathrm{m}$.

a)

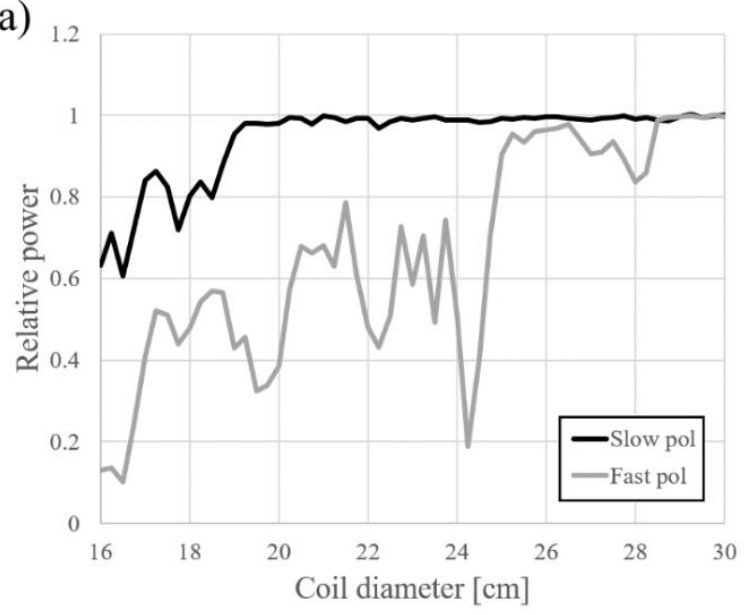

b)

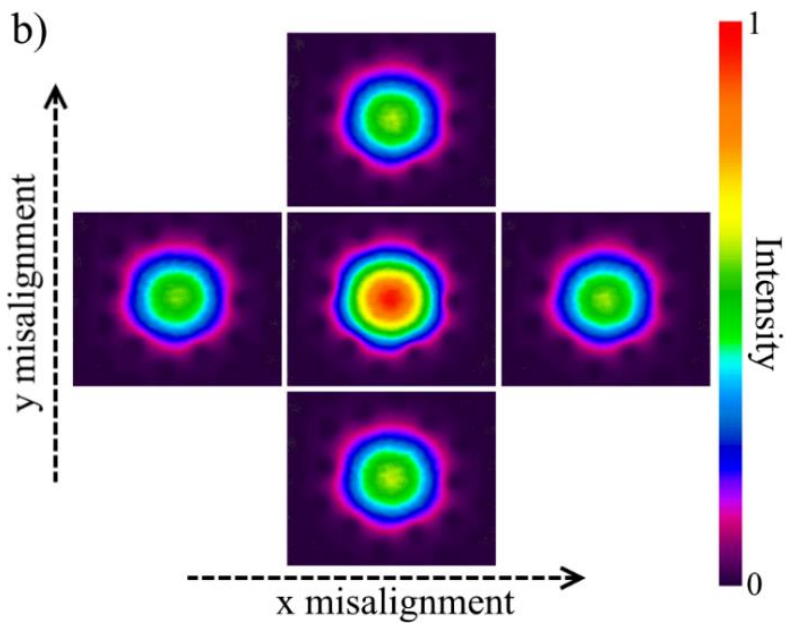

Figure 4: a) Measurement of the bending loss of the FM both polarized in the slow and fast axis of the fiber. b) Misalignment test performed with a coil diameter of $22 \mathrm{~cm}$.

\section{MONOLITHIC AMPLIFIER}

The low splice loss and single-mode operation of the fiber indicates efficient implementation of the fiber in a monolithic fiber amplifier system. A monolithic fiber amplifier has been build using the all-solid LMA PCF. A sketch of the amplifier system can be seen in figure 5. A pulsed seed laser was spliced to an isolator to protect the seed and 
measure the reflected light from the system. The output of the isolator was connected to a combiner, which transmitted the seed light in the core of a DC step-index fiber and the pump laser diodes (LD) into the cladding. The output fiber of the combiner was a DC few mode LMA SIF passive fiber which was spliced to the all-solid active fiber using the same splice configuration as for the splice tests.

The pulsed seed laser had a pulse length of $20 \mathrm{ps}$ and a repetition rate of $80 \mathrm{MHz}$ with a center wavelength of $1032 \mathrm{~nm}$ and a full-width-half-maximum (FWHM) of $1 \mathrm{~nm}$. The seed power at the start of the active fiber was approximately 1 W. The continuous wave non-wavelength-locked pump laser diodes (LD) had a FWHM of $4 \mathrm{~nm}$ and both the LDs and the combiner were water-cooled. The chiller temperature was set to achieve a center wavelength of the LDs of $976 \mathrm{~nm}$ for maximum operating current. For low operating currents, the center wavelength of the LDs was approximately 960 $\mathrm{nm}$. At maximum operating current the pump power at the input of the active fiber was $70 \mathrm{~W}$.

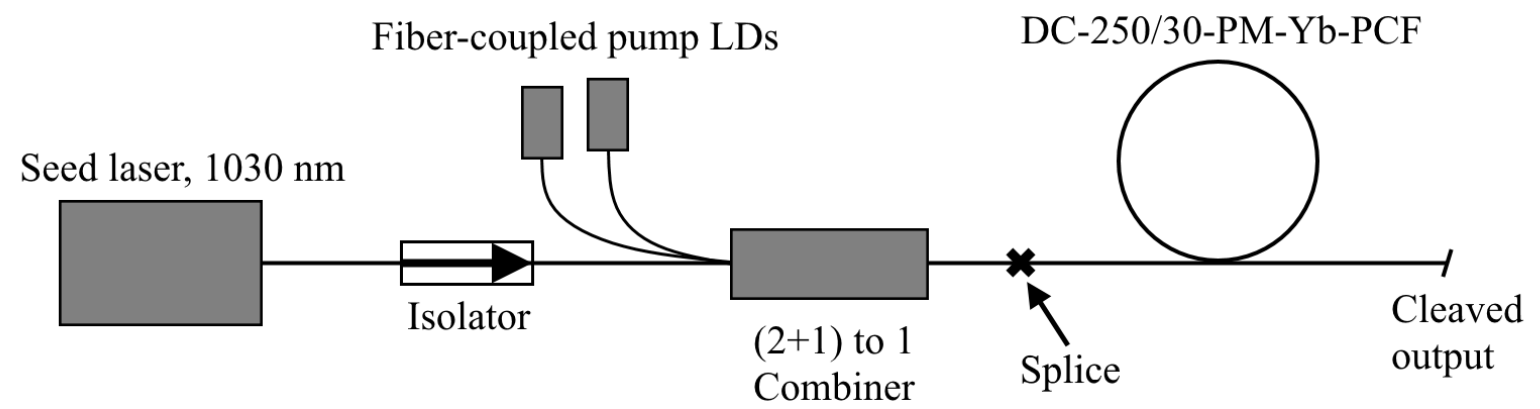

Figure 5: Sketch of the monolithic amplifier system. An Agiltron Inc. high power optical fiber isolator and an ITF $(2+1) \times 1$ combiner are used for the setup.

The monolithic amplifier was characterized using a cutback measurement and for each fiber length the signal power and PER were measured as a function of pump power while the spatial mode profile and spectrum were measured at maximum pump power. The optimal fiber length considering signal power was found to $2.65 \mathrm{~m}$. The signal power as a function of pump power and the spectrum at maximum signal power for the optimal fiber length can be seen in figure 6 . Since the wavelength of the pump depends on operating current, the slope efficiency exceeds 1 due to the pump entering the absorption peak of $\mathrm{Yb}$. The maximum signal power was measured to $43 \mathrm{~W}$ for an input pump power of $70.5 \mathrm{~W}$, which corresponds to an optical to optical efficiency of $61 \%$.

The spectrum found in figure 6 shows a peak with a FWHM of a few $\mathrm{nm}$ around the seed wavelength and no signs of non-linear effects. The broad feature around $1040 \mathrm{~nm}$ is the amplified spontaneous emission of the inverted $\mathrm{Yb}$ ions. The peak of the ASE is shifted due to higher absorption cross sections closer to $976 \mathrm{~nm}$. The spectrum shows that nonlinear effects in the fiber do not limit the signal power for the system. 


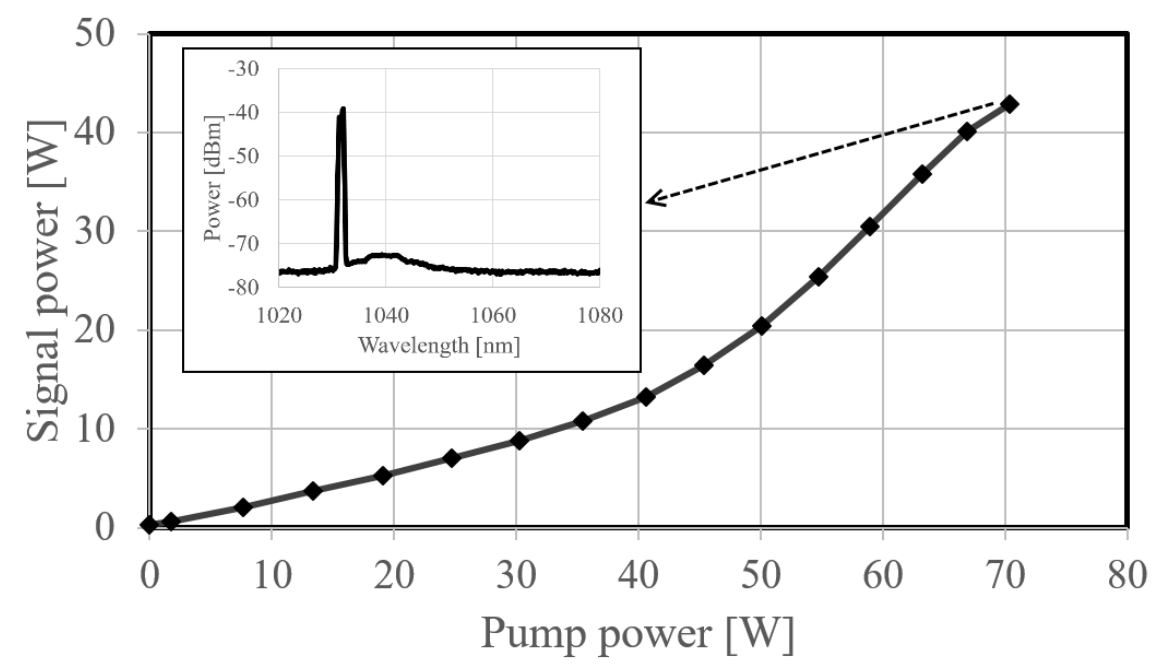

Figure 6: Signal power as a function of pump power and spectrum at maximum signal power for a fiber length of $2.65 \mathrm{~m}$.

In figure 7 the PER as a function of signal power for the optimal fiber length of $2.65 \mathrm{~m}$ and spatial field distribution at different signal powers are shown. The PER is above $18 \mathrm{~dB}$ for all signal powers and the spatial field distribution shows excellent beam quality with no signs of amplification of HOM. The good spectral, spatial, and polarization properties of the signal light are important for many applications of high power fiber amplifiers since conditions on the delivering system are relaxed. The excellent properties of the output signal light also show that the signal power is limited by pump power.

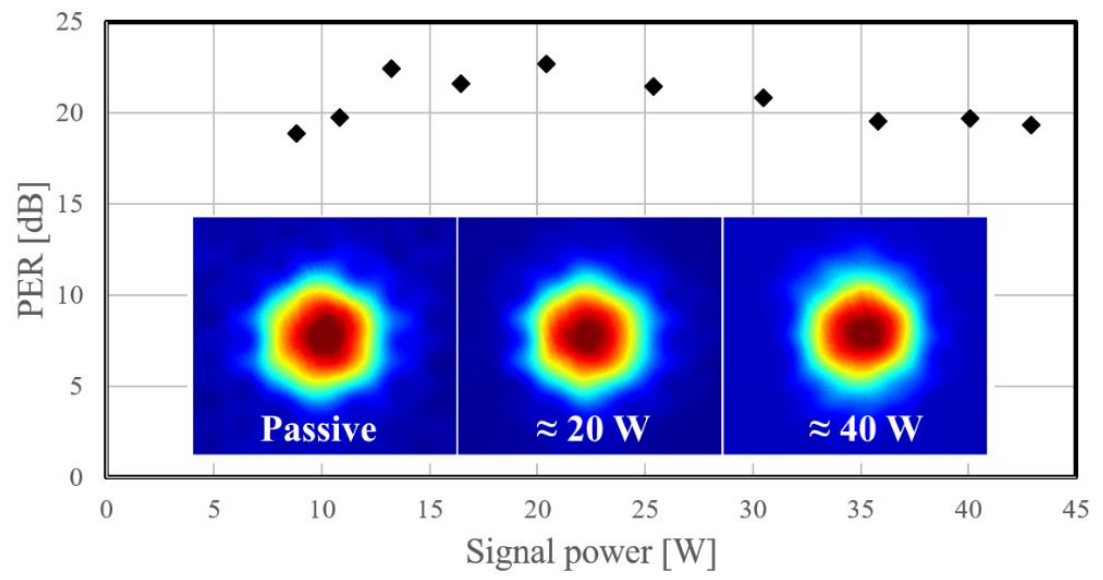

Figure 7: PER as a function of signal power for the optimal fiber length of $2.65 \mathrm{~m}$ and mode profiles during an active test.

For all fiber lengths, the properties of the light were similar to the presented data for the optimal length. The optimal length of the fiber was solely chosen by the magnitude of output power, which is proportional to the optical to optical efficiency. Figure 8 shows the optical to optical efficiency as a function of the fiber length. For short fiber lengths, the signal does not have the length to extract all the pump, while for longer fiber lengths, the absorption of $\mathrm{Yb}$ at the signal wavelength surpasses the gain from the pump. The optimum is found for a fiber length of $2.65 \mathrm{~m}$ with an efficiency of $61 \%$. The fiber has previously been tested in a free-space fiber amplifier setup achieving an optical to optical efficiency of $80 \%$ [7]. The difference in efficiency is due to differences in the coupling of the pump of the two amplifier systems. 
In a free-space setup, the coupling of the pump can be optimized to increase excitation of cladding modes with an overlap with the core, which is not possible in a monolithic setup. For the monolithic amplifier tested in this work residual pump data showed that some fraction of the pump did not interact with the core. To increase pump scrambling a kidney coil [8] was tried, which did not result in a significant effect. Other pump scrambling methods were not implemented but could possibly have been used to increase the efficiency of the amplifier.

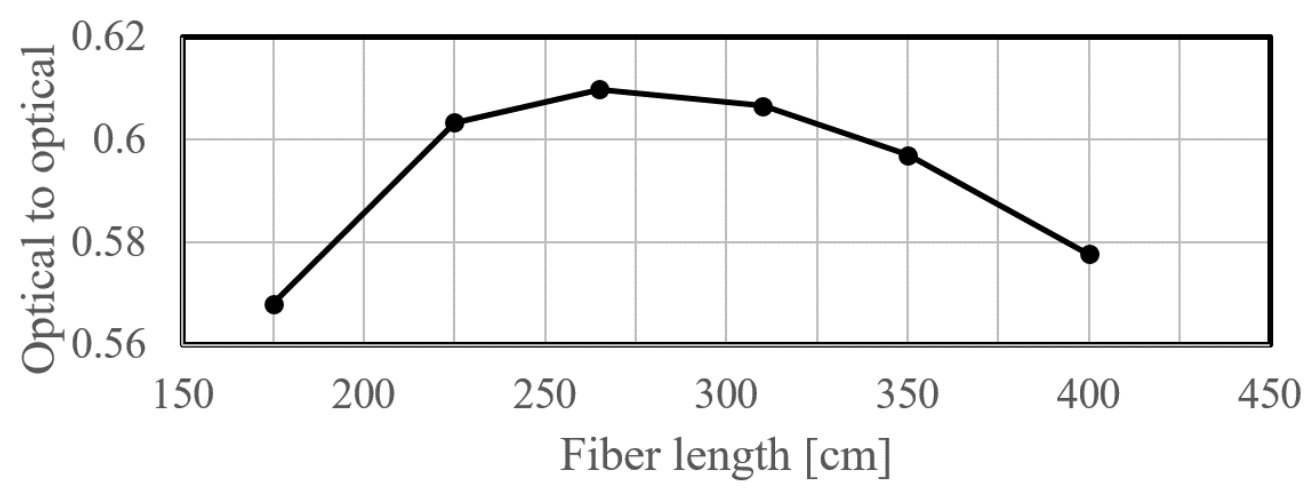

Figure 8: Optical to optical efficiency as a function of fiber length.

\section{Conclusion}

This work presents a monolithic fiber amplifier with an excellent spectral and spatial output light with a power of $43 \mathrm{~W}$ only limited by pump power. The active fiber used in the amplifier is a new all-solid single-mode photonic crystal fiber with a mode-field-diameter of $24 \mu \mathrm{m}$. Simple splicing of the all-solid PCF to itself was demonstrated achieving polarization maintaining splices and low splice losses below $0.2 \mathrm{~dB}$. The amplifier achieved optical to optical efficiencies of $61 \%$ using commercially available components and simple splice recipes.

\section{References}

[1] M. N. Zervas and C. A. Codemard, "High Power Fiber Lasers: A Review," in IEEE Journal of Selected Topics in Quantum Electronics, vol. 20, no. 5, pp. 219-241, Sept.-Oct. 2014, Art no. 0904123

[2] J. C. Knight, T. A. Birks, P. St. J. Russell, and D. M. Atkin. All-silica single-mode optical fiber with photonic crystal cladding. Opt. Lett., 21(19):1547-1549, Oct 1996

[3] Thomas Tanggaard Alkeskjold, Marko Laurila, Lara Scolari, and Jes Broeng, "Single-mode ytterbium-doped large-mode-area photonic bandgap rod fiber amplifier," Opt. Express 19, 7398-7409 (2011)

[4] Limin Xiao, M. S. Demokan, Wei Jin, Yiping Wang, and Chun-Liu Zhao, "Fusion Splicing Photonic Crystal Fibers and Conventional Single-Mode Fibers: Microhole Collapse Effect," J. Lightwave Technol. 25, 35633574 (2007)

[5] F. Luan, A. K. George, T. D. Hedley, G. J. Pearce, D. M. Bird, J. C. Knight, and P. St. J. Ru ssell, "All-solid photonic bandgap fiber," Opt. Lett. 29, 2369-2371 (2004)

[6] Guancheng Gu, Fanting Kong, Thomas Hawkins, Joshua Parsons, Maxwell Jones, Christopher Dunn, Monica T. Kalichevsky-Dong, Kunimasa Saitoh, and Liang Dong, "Ytterbium-doped large-mode-area all-solid photonic bandgap fiber lasers," Opt. Express 22, 13962-13968 (2014)

[7] Sidsel R. Papior et al., "Photonic crystal fiber technology for high-performance all-fiber monolithic ultrafast fiber amplifiers," Proc. SPIE 10512, Fiber Lasers XV: Technology and Systems, 1051212 (26 February 2018);

[8] Yahua Li, S. D. Jackson and S. Fleming, "High absorption and low splice loss properties of hexagonal doubleclad fiber," in IEEE Photonics Technology Letters, vol. 16, no. 11, pp. 2502-2504, Nov. 2004 